180

\section{IDENTIFICAZIONE MOLECOLARE RAPIDA DI BATTERI E DI GENI DI RESISTENZA DA FLACONI EMOCOLTURALI: ESPERIENZA PEDIATRICA}

Bernaschi P.', Manfredini C.', Lucignano B.', Ranno S.', Mancinelli L.', Russo C.', Menichella D.'

'U.O. di Microbiologia, Ospedale Pediatrico "Bambino Gesù" - Istituto di Ricovero e Cura a Carattere Scientifico, Piazza S. Onofrio 4, 00165 Roma

Introduzione. La diagnosi di batteriemia e/o sepsi rappresenta a tutt'oggi una sfida microbiologica in termini di accuratezza e rapidità nella identificazione dei germi responsabili di setticemie. I saggi fenotipici biochimici di identificazione e antibiotico-suscettibilità dei microrganismi isolati da flaconi emocolturali positivi prevedono un tempo di refertazione di circa 48 ore. Nel nostro studio, abbiamo valutato un nuovo test molecolare rapido, (GenoType Blood Cultures ${ }^{\circledR}$-Hain Lifescience) dichiarato in grado di identificare batteri e determinare la presenza dei geni di resistenza mecA, vanA/B/C. Razionale dello studio è stato quello di valutare l'applicazione del test su emocolture provenienti da pazienti pediatrici afferenti all'Ospedale "Bambino Gesù” e di determinare la riduzione del Turn Around Time (TAT) nella routine del laboratorio microbiologico clinico

Metodi. Da Settembre a Dicembre 2006 presso la U.O. di Microbiologia dell’Ospedale “Bambino Gesù” i flaconi PEDS PLUS/F Becton Dickinson) rilevati positivi allo strumento BACTEC 9240 sono stati contemporaneamente saggiati con metodo fenotipico (Vitek ${ }^{\circledast} 2$ - Biomerieux) e molecolare. L'identificazione genotipica GenoType BC è stata eseguita come da istruzioni del produttore. Le discrepanze identificative sono state risolte mediante analisi di sequenza genomica (16s rDNA).

Risultati. Sono state valutate 148 emocolture positive (103 cocchi Gram-positivi, 28 bacilli Gram-negativi isolati singolarmente e 14 infezioni miste).

Nella prima parte dello studio il sistema GenoType BC ha correttamente identificato 41/49 cocchi Gram positivi (Sensibilità 84\%) e 17/17 bacilli Gram negativi (Sensibilità 100\%). Dopo ottimizzazione "in house” dell'estrazione del DNA sono stati correttamente identificati 53/53 Cocchi Gram positivi e 11/11 bacilli Gram negativi (Sensibilità e Specificità 100\%). Sono state valutate inoltre 14 colture miste; di queste 1 è stata identificata in prima istanza solo dal sistema GenoType BC ( $P$. aeruginosa e $S$. mapltophilia) e successivamente dimostrata mediante subcoltura.

Conclusioni. Il test molecolare GenoType BC è risultato di facile e rapida esecuzione (TAT medio 5 ore), utile per la diagnosi rapida di setticemia nel paziente pediatrico.

\section{1}

\section{IL SEQUENZIAMENTO GENICO DEL I 6S rDNA NELLA DIAGNOSI DELLE INFEZIONI DA BATTERI ANAEROBI}

\author{
Coltella L., Ranno S., Argentieri M., Russo C., Menichella D. \\ U.O. Microbiologia, Ospedale Pediatrico Bambino Gesù, \\ Piazza Sant'Onofrio 4, 00165 Roma
}

Introduzione. I batteri anaerobi sono responsabili di infezioni spesso gravi riscontrabili in diversi siti corporei. L'identificazione attualmente è eseguita con metodi fenotipici, lunghi, laboriosi e non attuabili in tutti i laboratori.

La conseguenza è una sottostima dell’incidenza di tali batteri nella pratica clinica.

In questo studio abbiamo valutato l'impatto del sequenziamento di 500bp del gene 16S rDNA nella nostra diagnostica di routine di laboratorio.

Metodi. 152 ceppi di batteri anaerobi, isolati da campioni clinici cosecutivamente raccolti da pazienti pediatrici afferenti all'Ospedale Pediatrico Bambino Gesù, sono stati valutati sia con metodi fenotipici (esame morfologico, saggio biochimico miniaturizzato) che con il metodo genotipico del sequenziamento di 500bp del gene 16S rDNA.

Risultati. il 56\% dei ceppi in esame ha mostrato piena concordanza identificativa fra i due metodi, fornendo la stessa identificazione; per il $20 \%$ dei ceppi la concordanza si è limitata a livello di genere, mentre a livello di specie solo il metodo molecolare è stato in grado di fornire un'identificazione. Nell'11\% dei ceppi i due sistemi non hanno mostrato concordanza ne a livello di genere ne di specie.

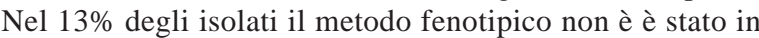
grado di identificare il microrganismo anaerobio in esame. Se si analizzano in particolare tre gruppi di batteri anaerobi: i principali patogeni (B. fragilis group, $C$. difficile e Fusobacterium); il germe endogeno di più frequente isolamento (P. acnes); e altri batteri che hanno un ruolo nell'infezione quali: (Peptostreptococcus, Prevotella, Porphyromonas, Veillonella).

Conclusioni. I nostri risultati confermano che i metodi fenotipici non sono sufficienti nella diagnostica delle infezioni da batteri anaerobi: infatti $P$. acnes risulta l'unico microrganismo anaerobio sempre correttamente identificato dai metodi fenotipici.

Al contrario, il sequenziamento genico rappresenta un valido aiuto nella pratica di laboratorio, permettendo una corretta identificazione dei patogeni anaerobi non soltanto a livello di genere, ma anche a livello di specie. 